\title{
Existence of solutions for a coupled system of Caputo-Hadamard type fractional differential equations with Hadamard fractional integral conditions
}

\author{
Mohamed Houas ${ }^{\mathrm{a}}$ \\ ${ }^{a}$ Department of Mathematics, UDBKM, Khemis Miliana University, Khemis Miliana, Algeria.
}

\begin{abstract}
In this work, we study existence and uniqueness of solutions for a coupled system of nonlinear fractional differential equations involving two Caputo-Hadamard-type fractional derivatives. By applying the Banach's fixed point theorem and Shaefer's fixed point theorem, the existence of solutions is obtained.The results obtained in this work are well illustrated with the aid of examples.
\end{abstract}

Keywords: Caputo-Hadamard fractional calculus, Existence, Boundary conditions, Fixed point theorem.

2010 MSC: 34A08, 34B10,34k05.

\section{Introduction and Preliminaries}

The fractional-type differential equations are generalizations of classical integer order differential equations and they are increasingly used to model problems in finance, fluid dynamics, biology and other areas of application. Recently, many studies on fractional differential equations, involving fractional derivative operators such as Riemann-Liouville fractional derivative [11, 20], Caputo fractional derivative [3, 5, 7], Hadamard fractional derivative [10, 18, 19, and Caputo-Hadamard fractional derivative [2, 4, 6], have appeared during

Email address: m.houas.st@univ-dbkm.dz (Mohamed Houas) 
the past several years. Moreover, by applying a variety of fixed point theorems (such as Banach contraction principle, Krasnoselskii's fixed point theorem, Schaefers fixe point theorem, Leray-Schauder's nonlinear alternative and Leray-Schauder's degree theory) several scholars presented the existence results for various classes of fractional differential equations, see for example [5, 6, 11, 15, 17] and the references cited therein. The study of coupled systems of fractional differential equations is also very significant as such systems appear in a variety of problems of applied nature, see [1, 8, 13, 14]. Some recent works on coupled systems of fractional differential equations with different fractional derivative operators can be found in [1, 9, 17, 21] and the references cited therein. In this paper, we consider a coupled system of Caputo-Hadamard-type fractional differential equations

$$
\left\{\begin{array}{c}
D^{\beta_{1}}\left(D^{\alpha_{1}}+\lambda_{1}\right) u(t)-f(t, u(t), v(t))=I^{\theta_{1}} \varphi(t, u(t), v(t)), t \in[1, e] \\
0<\beta_{1}, \alpha_{1} \leq 1,1<\beta_{1}+\alpha_{1} \leq 2, \quad \theta_{1}>0 \\
D^{\beta_{2}}\left(D^{\alpha_{2}}+\lambda_{2}\right) v(t)-g(t, u(t), v(t))=I^{\theta_{2}} \psi(t, u(t), v(t)), t \in[1, e] \\
0<\beta_{2}, \alpha_{2} \leq 1,1<\beta_{2}+\alpha_{2} \leq 2, \quad \theta_{2}>0
\end{array}\right.
$$

supplemented with nonlocal Hadamard fractional integral conditions

$$
\left\{\begin{array}{l}
a I^{p_{1}} u\left(\eta_{1}\right)=\gamma_{1}, b I^{q_{1}} x\left(\xi_{1}\right)=\gamma_{2}, p_{1}, q_{1}>0,1<\eta_{1}, \xi_{1}<e, a, b \in \mathbb{R} \\
c I^{p_{2}} v\left(\eta_{2}\right)=\sigma_{1}, d I^{q_{2}} v\left(\xi_{2}\right)=\sigma_{2}, p_{2}, q_{2}>0,1<\eta_{2}, \xi_{2}<e, c, d \in \mathbb{R}
\end{array}\right.
$$

where $f, g, \varphi$ and $\psi:[1, e] \times \mathbb{R}^{2} \rightarrow \mathbb{R}$ are given continuous functions, $I^{\theta_{i}}, I^{p_{i}}, I^{q_{i}}$ are the Hadamard fractional integrals and $D^{\alpha_{i}}, D^{\beta_{i}}(i=1,2)$ are the Caputo-Hadamard fractional derivatives. The Hadamard fractional integral [1, 12, 16] of order $\alpha$ for a continuous function $\varphi:[1,+\infty) \rightarrow \mathbb{R}$ is defined by

$$
{ }_{H} I^{\alpha} \varphi(t)=\frac{1}{\Gamma(\alpha)} \int_{1}^{t}\left(\log \frac{t}{s}\right)^{\alpha-1} \frac{\varphi(s)}{s} d s, \alpha>0,
$$

with $\Gamma(\alpha)=\int_{0}^{\infty} e^{u} u^{\alpha-1} d u$. The Caputo-Hadamard fractional derivative [2, 12, 16] of order $\alpha$ for a continuous function $\varphi:[1,+\infty) \rightarrow \mathbb{R}$ is defined by

$$
{ }_{H}^{C} D^{\alpha} \varphi(t)=\frac{1}{\Gamma(n-\alpha)} \int_{1}^{t}\left(\log \frac{t}{s}\right)^{n-\alpha-1} \delta^{n} \varphi(s) \frac{d s}{s}={ }_{H} I^{n-\alpha}\left(\delta^{n} \varphi\right)(t),
$$

where $n=[\alpha]+1$ and $\delta^{n}=\left(t \frac{d}{d t}\right)^{n}$.

Lemma 1.1. [2, 12, 16] Let $x(t) \in A C_{\delta}^{n}[a, b]$ where $0<a<b<\infty$ and $A C_{\delta}^{n}[a, b]=\left\{g:[a, b] \rightarrow \mathbb{C}, \delta^{n-1} g(t) \in A C[a\right.$ We define

$$
I^{\rho} D^{\rho} x(t)=x(t)+\sum_{i=0}^{n-1} c_{i}(\log t)^{i}
$$

where $c_{i} \in R, i=1,2, \ldots, n-1$ and $n=[\rho]+1$.

Lemma 1.2. [15, 17] Let $S$ be a Banach space. Assume that $\mathcal{A}: \mathcal{S} \rightarrow \mathcal{S}$ is a completely continuous operator and that the set $\Lambda=\{x \in S: x=\sigma \mathcal{A}(x), 0<\sigma<1\}$ is bounded. Then, $\mathcal{A}$ has a fixed point in $S$.

We prove the following auxiliary lemma.

Lemma 1.3. Let $\Pi_{1} \neq 0$ and $h_{1} \in C([1, e], \mathbb{R})$. Then the solution of the problem

$$
D^{\beta_{1}}\left(D^{\alpha_{1}}+\lambda_{1}\right) u(t)=h_{1}(t), t \in[1, e], 0<\beta_{1}, \alpha_{1} \leq 1,1<\beta_{1}+\alpha_{1} \leq 2,
$$

with the Hadamard fractional integral conditions

$$
a I^{p_{1}} u\left(\eta_{1}\right)=\gamma, b I^{q_{1}} u\left(\xi_{1}\right)=\gamma, 1<\eta_{1}, \xi_{1}<e, a, b \in \mathbb{R},
$$


is given by

$$
\begin{aligned}
& u(t)=I^{\alpha_{1}+\beta_{1}} h_{1}(t)-\lambda_{1} I^{\alpha_{1}} u(t) \\
& +\frac{\Lambda_{4}(\log t)^{\alpha_{1}}-\Lambda_{3} \Gamma\left(\alpha_{1}+1\right)}{\Pi_{1} \Gamma\left(\alpha_{1}+1\right)}\left(\gamma_{1}-a I^{p_{1}+\alpha_{1}+\beta_{1}} h_{1}\left(\eta_{1}\right)+\lambda_{1} a I^{p_{1}+\alpha_{1}} u\left(\eta_{1}\right)\right) \\
& -\frac{\Lambda_{2}(\log t)^{\alpha_{1}}-\Lambda_{1} \Gamma\left(\alpha_{1}+1\right)}{\Pi_{1} \Gamma\left(\alpha_{1}+1\right)}\left(\gamma_{2}-b I^{q_{1}+\alpha_{1}+\beta_{1}} h_{1}\left(\xi_{1}\right)+\lambda_{1} b I^{q_{1}+\alpha_{1}} u\left(\xi_{1}\right)\right),
\end{aligned}
$$

where

$$
\begin{aligned}
\Pi_{1} & =\Lambda_{1} \Lambda_{4}-\Lambda_{2} \Lambda_{3} \neq 0, \\
\Lambda_{1} & =\frac{a\left(\log \eta_{1}\right)^{p_{1}+\alpha_{1}}}{\Gamma\left(p_{1}+\alpha_{1}+1\right)}, \quad \Lambda_{2}=\frac{a\left(\log \eta_{1}\right)^{p_{1}}}{\Gamma\left(p_{1}+1\right)}, \\
\Lambda_{3} & =\frac{b\left(\log \xi_{1}\right)^{q_{1}+\alpha_{1}}}{\Gamma\left(q_{1}+\alpha_{1}+1\right)}, \quad \Lambda_{4}=\frac{b\left(\log \xi_{1}\right)^{q_{1}}}{\Gamma\left(q_{1}+1\right)},
\end{aligned}
$$

Proof. Using Lemma 1.1, we can write

$$
u(t)=I^{\alpha+\beta} h_{1}(t)-\lambda_{1} I^{\alpha_{1}} u(t)+e_{0} \frac{1}{\Gamma\left(\alpha_{1}+1\right)}(\log t)^{\alpha_{1}}+e_{1},
$$

where $e_{0}$ and $e_{1}$ are arbitrary constants. By taking the Hadamard fractional integral of order $p_{1}$ for (6), we have

$$
I^{p_{1}} u(t)=I^{p_{1}+\alpha_{1}+\beta_{1}} h_{1}(t)-\lambda_{1} I^{p_{1}+\alpha_{1}} u(t)+e_{0} \frac{(\log t)^{p_{1}+\alpha_{1}}}{\Gamma\left(p_{1}+\alpha_{1}+1\right)}+e_{1} \frac{(\log t)^{p_{1}}}{\Gamma\left(p_{1}+1\right)} .
$$

Now we multiply (7) by $a$ and in particular, for $t=\eta_{1}$, we obtain

$$
\begin{aligned}
a I^{p_{1}} x\left(\eta_{1}\right)= & a I^{p_{1}+\alpha_{1}+\beta_{1}} \varphi\left(\eta_{1}\right)-a \lambda_{1} I^{p_{1}+\alpha_{1}} x\left(\eta_{1}\right) \\
& +a e_{0} \frac{(\log t)^{p_{1}+\alpha_{1}}}{\Gamma\left(p_{1}+\alpha_{1}+1\right)}+a e_{1} \frac{(\log t)^{p_{1}}}{\Gamma\left(p_{1}+1\right)} .
\end{aligned}
$$

Using the boundary conditions (4), we find that

$$
e_{0} \Lambda_{1}+e_{1} \Lambda_{2}=\gamma_{1}-a I^{p_{1}+\alpha_{1}+\beta_{1}} \varphi\left(\eta_{1}\right)+a \lambda_{1} I^{p_{1}+\alpha_{1}} u\left(\eta_{1}\right),
$$

and

$$
e_{0} \Lambda_{3}+e_{1} \Lambda_{4}=\gamma_{2}-b I^{q_{1}+\alpha_{1}+\beta_{1}} \varphi\left(\xi_{1}\right)+b \lambda_{1} I^{q_{1}+\alpha_{1}} u\left(\xi_{1}\right) .
$$

Solving (9) and $(10)$ for $e_{0}, e_{1}$, we get

$$
\begin{aligned}
e_{0}= & \frac{\Lambda_{4}}{\Pi_{1}}\left[\gamma_{1}-a I^{p_{1}+\alpha_{1}+\beta_{1}} \varphi\left(\eta_{1}\right)+a \lambda_{1} I^{p_{1}+\alpha_{1}} u\left(\eta_{1}\right)\right] \\
& -\frac{\Lambda_{4}}{\Pi_{1}}\left[\gamma_{2}-b I^{q_{1}+\alpha_{1}+\beta_{1}} \varphi\left(\xi_{1}\right)+b \lambda_{1} I^{q_{1}+\alpha_{1}} u\left(\xi_{1}\right)\right],
\end{aligned}
$$

and

$$
\begin{aligned}
e_{1}= & \frac{\Lambda_{3}}{\Pi_{1}}\left[\gamma_{2}-b I^{q_{1}+\alpha_{1}+\beta_{1}} \varphi\left(\xi_{1}\right)+b \lambda_{1} I^{q_{1}+\alpha_{1}} u\left(\xi_{1}\right)\right] \\
& -\frac{\Lambda_{1}}{\Pi_{1}}\left[\gamma_{1}-a I^{p_{1}+\alpha_{1}+\beta_{1}} \varphi\left(\eta_{1}\right)+a \lambda_{1} I^{p_{1}+\alpha_{1}} u\left(\eta_{1}\right)\right] .
\end{aligned}
$$

By substituting the value of $e_{0}$ and $e_{1}$ in (6), we obtain the solution (5). 
Lemma 1.4. Let $\Pi_{2} \neq 0$ and $h_{2} \in C([1, e], \mathbb{R})$. Then the solution of the problem

$$
\left\{\begin{array}{c}
D^{\beta_{2}}\left(D^{\alpha_{2}}+\lambda_{2}\right) v(t)=h_{2}(t), t \in[1, e], 0<\beta_{2}, \alpha_{2} \leq 1,1<\beta_{2}+\alpha_{2} \leq 2 \\
c I^{p_{2}} v\left(\eta_{2}\right)=\sigma_{1}, d I^{q_{2}} v\left(\xi_{2}\right)=\sigma_{2}, 1<\eta_{2}, \xi_{2}<e, c, d \in \mathbb{R},
\end{array}\right.
$$

is given by

$$
\begin{aligned}
& v(t)=I^{\alpha_{2}+\beta_{2}} h_{2}(t)-\lambda_{2} I^{\alpha_{2}} v(t) \\
& +\frac{\Delta_{2}(\log t)^{\alpha_{2}}-\Delta_{1} \Gamma\left(\alpha_{2}+1\right)}{\Pi_{2} \Gamma\left(\alpha_{2}+1\right)}\left(\sigma_{1}-c I^{p_{2}+\alpha_{2}+\beta_{2}} h_{2}\left(\eta_{2}\right)+\lambda_{2} c I^{p_{2}+\alpha_{2}} v\left(\eta_{2}\right)\right) \\
& -\frac{\Delta_{4}(\log t)^{\alpha_{2}}-\Delta_{3} \Gamma\left(\alpha_{2}+1\right)}{\Pi_{2} \Gamma\left(\alpha_{2}+1\right)}\left(\sigma_{2}-d I^{q_{2}+\alpha_{2}+\beta_{2}} h_{2}\left(\xi_{2}\right)+\lambda_{2} d I^{q_{2}+\alpha_{2}} v\left(\xi_{2}\right)\right),
\end{aligned}
$$

where

$$
\begin{array}{ll}
\Delta_{1}=\frac{c\left(\log \eta_{2}\right)^{p_{2}+\alpha_{2}}}{\Gamma\left(p_{2}+\alpha_{2}+1\right)}, & \Delta_{2}=\frac{c\left(\log \eta_{2}\right)^{p_{2}}}{\Gamma\left(p_{2}+1\right)}, \\
\Delta_{3}=\frac{d\left(\log \xi_{2}\right)^{q_{2}+\alpha_{2}}}{\Gamma\left(q_{2}+\alpha_{2}+1\right)}, & \Delta_{4}=\frac{d\left(\log \xi_{2}\right)^{q_{2}}}{\Gamma\left(q_{2}+1\right)},
\end{array}
$$

and

$$
\Pi_{2}=\Delta_{1} \Delta_{4}-\Delta_{2} \Delta_{3}, \quad \Pi_{2} \neq 0
$$

Proof. The proof it is similar to that of Lemma 1.4.

Let us now introduce the space

$W=\{(u, v): u, v \in C([1, T], \mathbb{R})\}$ endowed with the norm $\left.\|u, v\|_{W}=\|u\|+\|v\|\right\}$, where

$$
\|u\|=\sup \{|u(t)|, t \in[1, T]\},\|v\|=\sup \{|v(t)|, t \in[1, T]\}
$$

It is clear that $\left(W,\|\cdot\|_{W}\right)$ is a Banach space. Throughout this paper, for convenience, the expressions $I^{\omega} \phi(s, u(s), v(s))(t)$, and $I^{\varpi} z(s)(t)$ mean

$$
\begin{aligned}
I^{\omega} \phi(s, u(s), v(s))(t) & =\frac{1}{\Gamma(\omega)} \int_{1}^{t}\left(\log \frac{t}{s}\right)^{\omega-1} \phi(s, u(s), u(s)) \frac{d s}{s} . \\
I^{\omega} z(s)(t) & =\frac{1}{\Gamma(\omega)} \int_{1}^{t}\left(\log \frac{t}{s}\right)^{\omega-1} z(s) \frac{d s}{s} .
\end{aligned}
$$

\section{Existence and uniqueness result}

In view of Lemma 1.3 and Lemma 1.4 , we define the operator $H: W \rightarrow W$ by:

$$
H(u, v)(t)=\left(\begin{array}{c}
H_{1}(u, v)(t) \\
H_{2}(u, v)(t)
\end{array}\right), t \in[1, e]
$$

where

$$
\begin{aligned}
& H_{1}(u, v)(t) \\
= & I^{\alpha_{1}+\beta_{1}} f(s, u(s), v(s))(t)+I^{\alpha_{1}+\beta_{1}+\theta_{1}} h_{1}(s, u(s), v(s))(t)-\lambda_{1} I^{\alpha_{1}} u(s)(t) \\
& +\frac{\Lambda_{2}(\log t)^{\alpha_{1}}-\Lambda_{1} \Gamma\left(\alpha_{1}+1\right)}{\Pi_{1} \Gamma\left(\alpha_{1}+1\right)}\left[\gamma_{1}-a I^{p_{1}+\alpha_{1}+\beta_{1}} f(s, x(s), y(s))\left(\eta_{1}\right)\right. \\
& \left.-a I^{p_{1}+\alpha_{1}+\beta_{1}+\theta_{1}} \varphi(s, u(s), v(s))\left(\eta_{1}\right)+\lambda_{1} a I^{p_{1}+\alpha_{1}} u(s)\left(\eta_{1}\right)\right] \\
& -\frac{\Lambda_{4}(\log t)^{\alpha}-\Lambda_{3} \Gamma\left(\alpha_{1}+1\right)}{\Pi_{1} \Gamma\left(\alpha_{1}+1\right)}\left[\gamma_{2}-b I^{q_{1}+\alpha_{1}+\beta_{1}} f(s, u(s), v(s))\left(\xi_{1}\right)\right. \\
& \left.-b I^{q_{1}+\alpha_{1}+\beta_{1}+\theta_{1}} h_{1}(s, u(s), v(s))\left(\xi_{1}\right)+\lambda_{1} b I^{q_{1}+\alpha_{1}} u(s)\left(\xi_{1}\right)\right]
\end{aligned}
$$


and

$$
\begin{aligned}
& H_{2}(u, v)(t) \\
= & I^{\alpha_{2}+\beta_{2}} g(s, u(s), v(s))(t)+I^{\alpha_{2}+\beta_{2}+\theta_{2}} \psi(s, u(s), v(s))(t)-\lambda_{2} I^{\alpha_{2}} v(s)(t) \\
& +\frac{\Delta_{2}(\log t)^{\alpha_{2}}-\Delta_{1} \Gamma\left(\alpha_{2}+1\right)}{\Pi_{2} \Gamma\left(\alpha_{2}+1\right)}\left[\sigma_{1}-c I^{p_{2}+\alpha_{2}+\beta_{2}} g(s, u(s), v(s))\left(\eta_{2}\right)\right. \\
& \left.-c I^{p_{2}+\alpha_{2}+\beta_{2}+\theta_{2}} \psi(s, u(s), v(s))\left(\eta_{2}\right)+\lambda_{2} c I^{p_{2}+\alpha_{2}} v(s)\left(\eta_{2}\right)\right] \\
& -\frac{\Delta_{4}(\log t)^{\alpha_{2}}-\Delta_{3} \Gamma\left(\alpha_{2}+1\right)}{\Pi_{2} \Gamma\left(\alpha_{2}+1\right)}\left[\sigma_{2}-d I^{q_{2}+\alpha_{2}+\beta_{2}} g(s, u(s), v(s))\left(\xi_{2}\right)\right. \\
& \left.-d I^{q_{2}+\alpha_{2}+\beta_{2}+\theta_{2}} \psi(s, u(s), v(s))\left(\xi_{2}\right)+\lambda_{2} d I^{q_{2}+\alpha_{2}} v(s)\left(\xi_{2}\right)\right] .
\end{aligned}
$$

For the sake of convenience, we impose the following conditions:

$\left(C_{1}\right): f, \varphi:[1, e] \times \mathbb{R} \times \mathbb{R} \rightarrow \mathbb{R}$ are continuous functions and there exist constants $k_{1}>0, k_{2}>0$ shch that for all $t \in[1, e]$ and $u_{i}, v_{i} \in \mathbb{R}(i=1,2)$,

$$
\left|f\left(t, u_{1}, v_{1}\right)-f\left(t, u_{2}, v_{2}\right)\right| \leq k_{1}\left|u_{1}-u_{2}\right|+\left|v_{1}-v_{2}\right|,
$$

and

$$
\left|\varphi\left(t, u_{1}, v_{1}\right)-\varphi\left(t, u_{2}, v_{2}\right)\right| \leq k_{2}\left(\left|u_{1}-u_{2}\right|+\left|v_{1}-v_{2}\right|\right) .
$$

$\left(C_{2}\right): g, \psi:[1, e] \times \mathbb{R} \times \mathbb{R} \rightarrow \mathbb{R}$ are continuous functions and there exist constants $l_{1}>0, l_{2}>0$ shch that for all $t \in[1, e]$ and $u_{i}, v_{i} \in \mathbb{R}(i=1,2)$,

$$
\left|g\left(t, u_{1}, v_{1}\right)-g\left(t, u_{2}, v_{2}\right)\right| \leq l_{1}\left(\left|u_{1}-u_{2}\right|+\left|v_{1}-v_{2}\right|\right) .
$$

and

$$
\left|\psi\left(t, u_{1}, v_{1}\right)-\psi\left(t, u_{2}, v_{2}\right)\right| \leq l_{2}\left(\left|u_{1}-u_{2}\right|+\left|v_{1}-v_{2}\right|\right) .
$$

We also introduce the following quantities:

$$
\begin{aligned}
\nabla_{1}: & =\frac{1}{\Gamma\left(\alpha_{1}+\beta_{1}+1\right)}+\frac{|a|\left[\left|\Lambda_{2}\right|+\left|\Lambda_{1}\right| \Gamma\left(\alpha_{1}+1\right)\right]\left(\log \eta_{1}\right)^{p_{1}+\alpha_{1}+\beta_{1}}}{\left|\Pi_{1}\right| \Gamma\left(\alpha_{1}+1\right) \Gamma\left(p_{1}+\alpha_{1}+\beta_{1}+1\right)} \\
& +\frac{|b|\left[\left|\Lambda_{4}\right|+\left|\Lambda_{3}\right| \Gamma\left(\alpha_{1}+1\right)\right]\left(\log \xi_{1}\right)^{q_{1}+\alpha_{1}+\beta_{1}}}{\left|\Pi_{1}\right| \Gamma\left(\alpha_{1}+1\right) \Gamma\left(q_{1}+\alpha_{1}+\beta_{1}+1\right)}, \\
\nabla_{2}: & =\frac{1}{\Gamma\left(\alpha_{1}+\beta_{1}+\theta_{1}+1\right)}+\frac{|a|\left[\left|\Lambda_{2}\right|+\left|\Lambda_{1}\right| \Gamma\left(\alpha_{1}+1\right)\right]\left(\log \eta_{1}\right)^{p_{1}+\alpha_{1}+\beta_{1}+\theta_{1}}}{\left|\Pi_{1}\right| \Gamma\left(\alpha_{1}+1\right) \Gamma\left(p_{1}+\alpha_{1}+\beta_{1}+\theta_{1}+1\right)} \\
& +\frac{|b|\left[\left|\Lambda_{4}\right|+\left|\Lambda_{3}\right| \Gamma\left(\alpha_{1}+1\right)\right]\left(\log \xi_{1}\right)^{q_{1}+\alpha_{1}+\beta_{1}+\theta_{1}}}{\left|\Pi_{1}\right| \Gamma\left(\alpha_{1}+1\right) \Gamma\left(q_{1}+\alpha_{1}+\beta_{1}+\theta_{1}+1\right)} \\
\nabla_{3}: & =\left|\lambda_{1}\right|\left[\frac{1}{\Gamma\left(\alpha_{1}+1\right)}+\frac{|a|\left[\left|\Lambda_{2}\right|+\left|\Lambda_{1}\right| \Gamma\left(\alpha_{1}+1\right)\right]\left(\log \eta_{1}\right)^{p_{1}+\alpha_{1}}}{\left|\Pi_{1}\right| \Gamma\left(\alpha_{1}+1\right) \Gamma\left(p_{1}+\alpha_{1}+1\right)},\right. \\
& \left.+\frac{\left.|b|\left(\left|\Lambda_{4}\right|+\left|\Lambda_{3}\right| \Gamma\left(\alpha_{1}+1\right)\right)\left(\log \xi_{1}\right)^{q_{1}+\alpha_{1}}\right]}{\left|\Pi_{1}\right| \Gamma\left(\alpha_{1}+1\right) \Gamma\left(q_{1}+\alpha_{1}+1\right)}\right] \\
\nabla_{4}: & =\frac{1}{\left|\Pi_{1}\right| \Gamma\left(\alpha_{1}+1\right)}\left[\left|\Lambda_{2}\right|+\left|\Lambda_{1}\right| \Gamma\left(\alpha_{1}+1\right)\left|\gamma_{1}\right|+\left|\Lambda_{4}\right|+\left|\Lambda_{3}\right| \Gamma\left(\alpha_{1}+1\right)\left|\gamma_{2}\right|\right] .
\end{aligned}
$$




$$
\begin{aligned}
\Phi_{1}: & =\frac{1}{\Gamma\left(\alpha_{2}+\beta_{2}+1\right)}+\frac{|c|\left[\left|\Delta_{2}\right|+\left|\Delta_{1}\right| \Gamma\left(\alpha_{2}+1\right)\right]\left(\log \eta_{2}\right)^{p_{2}+\alpha_{2}+\beta_{2}}}{\left|\Pi_{2}\right| \Gamma\left(\alpha_{2}+1\right) \Gamma\left(p_{2}+\alpha_{2}+\beta_{2}+1\right)} \\
& +\frac{|d|\left[\left|\Delta_{4}\right|+\left|\Delta_{3}\right| \Gamma\left(\alpha_{2}+1\right)\right]\left(\log \xi_{2}\right)^{q_{2}+\alpha_{2}+\beta_{2}}}{\left|\Pi_{2}\right| \Gamma\left(\alpha_{2}+1\right) \Gamma\left(q_{2}+\alpha_{2}+\beta_{2}+1\right)}, \\
\Phi_{2}: & =\frac{1}{\Gamma\left(\alpha_{2}+\beta_{2}+\theta_{2}+1\right)}+\frac{|c|\left[\left|\Delta_{2}\right|+\left|\Delta_{1}\right| \Gamma\left(\alpha_{2}+1\right)\right]\left(\log \eta_{2}\right)^{p_{2}+\alpha_{2}+\beta_{2}+\theta_{2}}}{\left|\Pi_{2}\right| \Gamma\left(\alpha_{2}+1\right) \Gamma\left(p_{2}+\alpha_{2}+\beta_{2}+\theta_{2}+1\right)} \\
& +\frac{|d|\left[\left|\Delta_{4}\right|+\left|\Delta_{3}\right| \Gamma\left(\alpha_{2}+1\right)\right]\left(\log \xi_{2}\right)^{q_{2}+\alpha_{2}+\beta_{2}+\theta_{2}}}{\left|\Pi_{2}\right| \Gamma\left(\alpha_{2}+1\right) \Gamma\left(q_{2}+\alpha_{2}+\beta_{2}+\theta_{2}+1\right)} \\
\Phi_{3}: & =\left|\lambda_{2}\right|\left[\frac{1}{\Gamma\left(\alpha_{2}+1\right)}+\frac{|c|\left[\left|\Delta_{2}\right|+\left|\Delta_{1}\right| \Gamma\left(\alpha_{2}+1\right)\right]\left(\log \eta_{1}\right)^{p_{2}+\alpha_{2}}}{\left|\Pi_{2}\right| \Gamma\left(\alpha_{2}+1\right) \Gamma\left(p_{2}+\alpha_{2}+1\right)},\right. \\
& \left.+\frac{\left.|d|\left(\left|\Delta_{4}\right|+\left|\Delta_{3}\right| \Gamma\left(\alpha_{2}+1\right)\right)\left(\log \xi_{2}\right)^{q_{2}+\alpha_{2}}\right]}{\left|\Pi_{2}\right| \Gamma\left(\alpha_{2}+1\right) \Gamma\left(q_{2}+\alpha_{2}+1\right)}\right], \\
\Phi_{4}: \quad & =\frac{1}{\left|\Pi_{1}\right| \Gamma\left(\alpha_{2}+1\right)}\left[\left|\Delta_{2}\right|+\left|\Delta_{1}\right| \Gamma\left(\alpha_{2}+1\right)\left|\sigma_{1}\right|+\left|\Delta_{4}\right|+\left|\Delta_{3}\right| \Gamma\left(\alpha_{2}+1\right)\left|\sigma_{2}\right|\right] .
\end{aligned}
$$

Theorem 2.1. Assume that conditions $\left(C_{1}\right)$ and $\left(C_{1}\right)$ hold and suppose that $\Pi_{1} \neq 0$ and $\Pi_{2} \neq 0$. If these inequalities

$$
k_{1} \nabla_{1}+k_{2} \nabla_{2}+\nabla_{3}<\frac{1}{2}, l_{1} \Phi_{1}+l_{2} \Phi_{2}+\Phi_{3}<\frac{1}{2},
$$

are valid, then system (1)-(2) has a unique solution.

Proof. Let us fix $\sup _{t \in[1, e]}|f(t, 0,0)|=L<\infty, \sup _{t \in[1, e]}|\varphi(t, 0,0)|=M<\infty$ and define

$$
\max \left[\frac{k_{1} \nabla_{1}+k_{2} \nabla_{2}+\nabla_{4}}{\frac{1}{2}-\left(L \nabla_{1}+M \nabla_{2}+\nabla_{3}\right)}, \frac{l_{1} \Phi_{1}+l_{2} \Phi_{2}+\Phi_{4}}{\frac{1}{2}-\left(L^{\prime} \Phi_{1}+M^{\prime} \Phi_{2}+\Phi_{3}\right)}\right] \leq r .
$$

We show that $H B_{r} \subset B_{r}$, where $B_{r}=\{(u, v) \in X \times Y:\|(u, v)\| \leq r\}$. For $(u, v) \in B_{r}$ and by $\left(H_{1}\right)$, we have

$$
\begin{aligned}
|f(t, u, v)| & \leq|f(t, u, v)-f(t, 0,0)|+|f(t, 0,0)| \leq k_{1}\|u\|+k_{1}\|v\|+L \\
& \leq k_{1}\|u, v\|_{W}+L \leq k_{1} r+L \\
|\varphi(t, u, v)| & \leq|\varphi(t, u, v)-\varphi(t, 0,0)|+|\varphi(t, 0,0)| \leq k_{2}\|u\|+k_{2}\|v\|+M \\
& \leq k_{2}\|u, v\|_{W}+M \leq k_{2} r+M,
\end{aligned}
$$

Similarly,we have

$$
\begin{aligned}
& |g(t, u, v)| \leq l_{1}\|u\|+l_{1}\|v\|+L^{\prime} \leq l_{1}\|u, v\|_{W}+L^{\prime} \leq l_{1} r+L^{\prime}, \\
& |\psi(t, u, v)| \leq l_{2}\|u\|+l_{2}\|v\|+M^{\prime} \leq l_{2}\|u, v\|_{W}+M^{\prime} \leq l_{2} r+M^{\prime},
\end{aligned}
$$


By 18), we can write

$$
\begin{aligned}
& \left\|H_{1}(u, v)\right\| \\
& \leq \sup _{t \in[1, e]}\left\{I^{\alpha_{1}+\beta_{1}}|f(s, u(s), v(s))|(t)+I^{\alpha_{1}+\beta_{1}+\theta_{1}}|\psi(s, u(s), v(s))|(t)+\left|\lambda_{1}\right| I^{\alpha_{1}}|v(s)|(t)\right. \\
& +\frac{\left|\Lambda_{2}\right|(\log t)^{\alpha_{1}}+\left|\Lambda_{1}\right| \Gamma\left(\alpha_{1}+1\right)}{\left|\Pi_{1}\right| \Gamma\left(\alpha_{1}+1\right)}\left(\left|\gamma_{1}\right|+|a| I^{p_{1}+\alpha_{1}+\beta_{1}}|f(s, u(s), v(s))|\left(\eta_{1}\right)\right. \\
& \left.+|a| I^{p_{1}+\alpha_{1}+\beta_{1}+\theta_{1}}|\varphi(s, u(s), v(s))|\left(\eta_{1}\right)+\left|\lambda_{1}\right|\left|a_{1}\right| I^{p_{1}+\alpha_{1}}|u(s)|\left(\eta_{1}\right)\right) \\
& +\frac{\left|\Lambda_{4}\right|(\log t)^{\alpha_{1}}+\left|\Lambda_{3}\right| \Gamma\left(\alpha_{1}+1\right)}{\left|\Pi_{1}\right| \Gamma\left(\alpha_{1}+1\right)}\left(\left|\gamma_{2}\right|+|b| I^{q_{1}+\alpha_{1}+\beta_{1}}\left|f_{1}(s, u(s), v(s))\right|\left(\xi_{1}\right)\right. \\
& \left.\left.+|b| I^{q_{1}+\alpha_{1}+\beta_{1}+\theta_{1}}|\varphi(s, u(s), v(s))|\left(\xi_{1}\right)+\left|\lambda_{1}\right||b| I^{q_{1}+\alpha_{1}}|u(s)|\left(\xi_{1}\right)\right)\right\} \\
& \leq\left[\frac{1}{\Gamma\left(\alpha_{1}+\beta_{1}+1\right)}+\frac{|a|\left(\left|\Lambda_{2}\right|+\left|\Lambda_{1}\right| \Gamma\left(\alpha_{1}+1\right)\right)\left(\log \eta_{1}\right)^{p_{1}+\alpha_{1}+\beta_{1}}}{\left|\Pi_{1}\right| \Gamma\left(\alpha_{1}+1\right) \Gamma\left(p_{1}+\alpha_{1}+\beta_{1}+1\right)}\right. \\
& \left.+\frac{|b|\left(\left|\Lambda_{4}\right|+\left|\Lambda_{3}\right| \Gamma\left(\alpha_{1}+1\right)\right)\left(\log \xi_{1}\right)^{q_{1}+\alpha_{1}+\beta_{1}}}{\left|\Pi_{1}\right| \Gamma\left(\alpha_{1}+1\right) \Gamma\left(q_{1}+\alpha_{1}+\beta_{1}+1\right)}\right]\left(L r+k_{1}\right) \\
& +\left[\frac{1}{\Gamma\left(\alpha_{1}+\beta_{1}+\theta_{1}+1\right)}+\frac{|a|\left(\left|\Lambda_{2}\right|+\left|\Lambda_{1}\right| \Gamma\left(\alpha_{1}+1\right)\right)\left(\log \eta_{1}\right)^{p_{1}+\alpha_{1}+\beta_{1}+\theta_{1}}}{\left|\Pi_{1}\right| \Gamma\left(\alpha_{1}+1\right) \Gamma\left(p_{1}+\alpha_{1}+\beta_{1}+\theta_{1}+1\right)}\right. \\
& \left.+\frac{|b|\left(\left|\Lambda_{4}\right|+\left|\Lambda_{3}\right| \Gamma\left(\alpha_{1}+1\right)\right)\left(\log \xi_{1}\right)^{q_{1}+\alpha_{1}+\beta_{1}+\theta_{1}}}{\left|\Pi_{1}\right| \Gamma\left(\alpha_{1}+1\right) \Gamma\left(q_{1}+\alpha_{1}+\beta_{1}+\theta_{1}+1\right)}\right]\left(L r+k_{2}\right) \\
& +\left|\lambda_{1}\right|\left[\frac{1}{\Gamma\left(\alpha_{1}+1\right)}+\frac{|a|\left(\left|\Lambda_{2}\right|+\left|\Lambda_{1}\right| \Gamma\left(\alpha_{1}+1\right)\right)\left(\log \eta_{1}\right)^{p_{1}+\alpha_{1}}}{\left|\Pi_{1}\right| \Gamma\left(\alpha_{1}+1\right) \Gamma\left(p_{1}+\alpha_{1}+1\right)}\right. \\
& \left.+\frac{\left|b_{1}\right|\left(\left|\Lambda_{4}\right|+\left|\Lambda_{3}\right| \Gamma\left(\alpha_{1}+1\right)\right)\left(\log \xi_{1}\right)^{q_{1}+\alpha_{1}}}{\left|\Pi_{1}\right| \Gamma\left(\alpha_{1}+1\right) \Gamma\left(q_{1}+\alpha_{1}+1\right)}\right] r \\
& +\frac{1}{\left|\Pi_{1}\right| \Gamma\left(\alpha_{1}+1\right)}\left[\left|\Delta_{2}\right|+\left|\Delta_{1}\right| \Gamma\left(\alpha_{1}+1\right)\left|\gamma_{1}\right|+\left|\Delta_{4}\right|+\left|\Delta_{3}\right| \Gamma\left(\alpha_{1}+1\right)\left|\gamma_{2}\right|\right] \\
& =\left(L r+k_{1}\right) \nabla_{1}+\left(M r+k_{2}\right) \nabla_{2}+\nabla_{3} r+\nabla_{4} \text {, }
\end{aligned}
$$

which implies that

$$
\left\|H_{1}(u, v)\right\| \leq\left(L \nabla_{1}+M \nabla_{2}+\nabla_{3}\right) r+k_{1} \nabla_{1}+k_{2} \nabla_{2}+\nabla_{4} \leq \frac{r}{2} .
$$

In a similar manner, using (19), we obtain

$$
\left\|H_{2}(u, v)\right\| \leq\left(L^{\prime} \Phi_{1}+M^{\prime} \Phi_{2}+\Phi_{3}\right) r+l_{1} \Phi_{1}+l_{2} \Phi_{2}+\Phi_{4} \leq \frac{r}{2} .
$$

From the definition of $\|\cdot\|_{W}$, we can write

$$
\|H(u, v)\|_{W}=\left\|H_{1}(u, v)\right\|+\left\|H_{2}(u, v)\right\| \leq r .
$$


Now, for $u_{i}, v_{i} \in B_{r}, i=1,2$, we have

$$
\begin{aligned}
& \left|H_{1}\left(u_{1}, v_{1}\right)-H_{1}\left(u_{2}, v_{2}\right)(t)\right| \\
& \leq \sup _{t \in[1, e]}\left\{I^{\alpha_{1}+\beta_{1}}\left|f\left(s, u_{1}(s), v_{1}(s)\right)-f\left(s, u_{2}(s), v_{2}(s)\right)\right|(t)\right. \\
& +\left|\lambda_{1}\right| I^{\alpha_{1}}\left|u_{1}(s)-u_{2}(s)\right|(t) \\
& +I^{\alpha_{1}+\beta_{1}+\theta_{1}}\left|\varphi\left(s, u_{1}(s), v_{1}(s)\right)-\varphi\left(s, u_{2}(s), v_{2}(s)\right)\right|(t) \\
& +\frac{\left|\Lambda_{2}\right|(\log t)^{\alpha_{1}}+\left|\Lambda_{1}\right| \Gamma\left(\alpha_{1}+1\right)}{\left|\Pi_{1}\right| \Gamma\left(\alpha_{1}+1\right)} \\
& \times\left[|a| I^{p_{1}+\alpha_{1}+\beta_{1}}\left|f\left(s, u_{1}(s), v_{1}(s)\right)-f\left(s, u_{2}(s), v_{2}(s)\right)\right|\left(\eta_{1}\right)\right. \\
& +|a| I^{p_{1}+\alpha_{1}+\beta_{1}+\theta_{1}}\left|\varphi\left(s, u_{1}(s), v_{1}(s)\right)-\varphi\left(s, u_{2}(s), v_{2}(s)\right)\right|\left(\eta_{1}\right) \\
& \left.+\left|\lambda_{1}\right||a| I^{p_{1}+\alpha_{1}}\left|u_{1}(s)-u_{2}(s)\right|\left(\eta_{1}\right)\right] \\
& +\frac{\left|\Lambda_{4}\right|(\log t)^{\alpha_{1}}+\left|\Lambda_{3}\right| \Gamma\left(\alpha_{1}+1\right)}{\left|\Pi_{1}\right| \Gamma\left(\alpha_{1}+1\right)} \\
& \times\left[|b| I^{q_{1}+\alpha_{1}+\beta_{1}}\left|f\left(s, u_{1}(s), v_{1}(s)\right)-f\left(s, u_{2}(s), v_{2}(s)\right)\right|\left(\xi_{1}\right)\right. \\
& +|b| I^{q_{1}+\alpha_{1}+\beta_{1}+\theta_{1}}\left|\varphi\left(s, u_{1}(s), v_{1}(s)\right)-\varphi\left(s, u_{2}(s), v_{2}(s)\right)\right|\left(\xi_{1}\right) \\
& \left.\left.+\left|\lambda_{1}\right||b| I^{q_{1}+\alpha_{1}}\left|u_{1}(s)-u_{2}(s)\right|\left(\xi_{1}\right)\right]\right\} \\
& \leq\left[\frac{1}{\Gamma\left(\alpha_{1}+\beta_{1}+1\right)}+\frac{|a|\left(\left|\Lambda_{2}\right|+\left|\Lambda_{1}\right| \Gamma\left(\alpha_{1}+1\right)\right)\left(\log \eta_{1}\right)^{p_{1}+\alpha_{1}+\beta_{1}}}{\left|\Pi_{1}\right| \Gamma\left(\alpha_{1}+1\right) \Gamma\left(p_{1}+\alpha_{1}+\beta_{1}+1\right)}\right. \\
& \left.+\frac{|b|\left(\left|\Lambda_{4}\right|+\left|\Lambda_{4}\right| \Gamma\left(\alpha_{1}+1\right)\right)\left(\log \xi_{1}\right)^{q_{1}+\alpha_{1}+\beta_{1}}}{\left|\Pi_{1}\right| \Gamma\left(\alpha_{1}+1\right) \Gamma\left(q_{1}+\alpha_{1}+\beta_{1}+1\right)}\right] k_{1}\left(\left\|u_{1}-u_{2}\right\|+\left\|v_{1}-v_{2}\right\|\right) \\
& +\left[\frac{1}{\Gamma\left(\alpha_{1}+\beta_{1}+\theta_{1}+1\right)}+\frac{|a|\left(\left|\Lambda_{2}\right|+\left|\Lambda_{1}\right| \Gamma\left(\alpha_{1}+1\right)\right)\left(\log \eta_{1}\right)^{p_{1}+\alpha_{1}+\beta_{1}+\theta_{1}}}{\left|\Pi_{1}\right| \Gamma\left(\alpha_{1}+1\right) \Gamma\left(p_{1}+\alpha_{1}+\beta_{1}+\theta_{1}+1\right)}\right. \\
& \left.+\frac{|b|\left(\left|\Lambda_{4}\right|+\left|\Lambda_{3}\right| \Gamma\left(\alpha_{1}+1\right)\right)\left(\log \xi_{1}\right)^{q_{1}+\alpha_{1}+\beta_{1}+\theta_{1}}}{\left|\Pi_{1}\right| \Gamma\left(\alpha_{1}+1\right) \Gamma\left(q_{1}+\alpha_{1}+\beta_{1}+\theta_{1}+1\right)}\right] k_{2}\left(\left\|u_{1}-u_{2}\right\|+\left\|v_{1}-v_{2}\right\|\right) \\
& +\left|\lambda_{1}\right|\left[\frac{1}{\Gamma\left(\alpha_{1}+1\right)}+\frac{|a|\left(\left|\Lambda_{2}\right|+\left|\Lambda_{1}\right| \Gamma\left(\alpha_{1}+1\right)\right)\left(\log \eta_{1}\right)^{p_{1}+\alpha_{1}}}{\left|\Pi_{1}\right| \Gamma\left(\alpha_{1}+1\right) \Gamma\left(p_{1}+\alpha_{1}+1\right)}\right. \\
& \left.+\frac{|b|\left(\left|\Lambda_{4}\right|+\left|\Lambda_{3}\right| \Gamma\left(\alpha_{1}+1\right)\right)\left(\log \xi_{1}\right)^{q_{1}+\alpha_{1}}}{\left|\Pi_{1}\right| \Gamma\left(\alpha_{1}+1\right) \Gamma\left(q_{1}+\alpha_{1}+1\right)}\right]\left\|u_{1}-u_{2}\right\| \\
& =\left[k_{1} \nabla_{1}+k_{2} \nabla_{2}+\nabla_{3}\right]\left(\left\|u_{1}-u_{2}\right\|+\left\|v_{1}-v_{2}\right\|\right) \text {, }
\end{aligned}
$$

and, consequently, we obtain

$$
\left\|H_{1}\left(u_{1}, v_{1}\right)-H_{1}\left(u_{2}, v_{2}\right)\right\| \leq\left[k_{1} \nabla_{1}+k_{2} \nabla_{2}+\nabla_{3}\right]\left(\left\|u_{1}-u_{2}\right\|+\left\|v_{1}-v_{2}\right\|\right) .
$$

Similarly, we can have

$$
\left\|H_{2}\left(u_{1}, v_{1}\right)-H_{2}\left(u_{2}, v_{2}\right)\right\| \leq\left[l_{1} \Phi_{1}+l_{2} \Phi_{2}+\Phi_{3}\right]\left(\left\|u_{1}-u_{2}\right\|+\left\|v_{1}-v_{2}\right\|\right) .
$$

Consequently, we obtain

$$
\begin{aligned}
& \left\|H\left(u_{1}, v_{1}\right)-H\left(u_{2}, v_{2}\right)\right\|_{W} \\
\leq & {\left[k_{1} \nabla_{1}+k_{2} \nabla_{2}+\nabla_{3}+l_{1} \Phi_{1}+l_{2} \Phi_{2}+\Phi_{3}\right]\left(\left\|u_{1}-u_{2}\right\|+\left\|v_{1}-v_{2}\right\|\right) . }
\end{aligned}
$$


Since $k_{1} \nabla_{1}+k_{2} \nabla_{2}+\nabla_{3}+l_{1} \Phi_{1}+l_{2} \Phi_{2}+\Phi_{3}<1$, therefore, $H$ is a contraction. So, by Banach's fixed point theorem, the operator $H$ has a unique fixed point, which is the unique solution of system (1)-(2). This completes the proof.

Example 2.1. Consider the following system

$$
\left\{\begin{array}{c}
D^{\frac{22}{31}}\left(D^{\frac{10}{13}}+\frac{2}{49}\right) u(t)-\frac{1}{\left(25 \pi^{2}+t\right)}(\sin u(t)-\cos v(t)) \\
=I^{\frac{3}{5}}\left[\frac{1}{55 t^{2}+1}\left(\frac{|u(t)|}{|u(t)|+1}+\frac{|v(t)|}{|v(t)|+1}\right)\right], t \in[1, e], \\
D^{\frac{11}{14}}\left(D^{\frac{8}{9}}+\frac{7}{80}\right) v(t)-\frac{1-e^{-t}}{57(t+1)^{2}}\left(\frac{|u(t)|}{2|u(t)|+1}+\frac{|v(t)|}{3|v(t)|+2}\right) \\
=I^{\frac{19}{22}}\left[\frac{1}{49\left(t^{2}+2\right)}(\cos u(t)+\cos v(t))\right], t \in[1, e], \\
I^{\frac{2}{3}} u\left(\frac{8}{5}\right)=(\log 8-\log 5)^{-\frac{1}{3}}, I^{\frac{57}{60}} u\left(\frac{7}{4}\right)=(\log 7-\log 4)^{-\frac{1}{3}} \\
\frac{1}{20} I^{\frac{3}{4}} v\left(\frac{e}{2}\right)=(1-\log 2)^{-\frac{1}{4}}, \frac{2}{35} I^{\frac{42}{51}} v(\sqrt{2})=\frac{2}{5}(\log 2)^{-\frac{9}{51}}
\end{array}\right.
$$

For this example, we have

$$
\begin{aligned}
f(t, u, v) & =\frac{1}{\left(25 \pi^{2}+t\right)}(\sin u-\cos v) \\
g(t, u, v) & =\frac{1-e^{-t}}{57(t+1)^{2}}\left(\frac{|u(t)|}{2|u|+1}+\frac{|v|}{3|v|+2}\right) \\
\varphi(t, u, v) & =\frac{1}{55 t^{2}+1}\left(\frac{|u|}{|u|+1}+\frac{|v|}{|v|+1}\right), \\
\psi(t, u, v) & =\frac{1}{49\left(t^{2}+2\right)}(\cos u+\cos v) .
\end{aligned}
$$

With the given data, we find that

$$
\begin{aligned}
& \Lambda_{1} \simeq-0.26587, \Lambda_{2} \simeq-0.66963, \Lambda_{3} \simeq 0.37963, \Lambda_{4} \simeq 0.58793 \\
& \Pi_{1}=\Lambda \Lambda_{4}-\Lambda_{2} \Lambda_{3} \simeq 0.097899 \\
& \nabla_{1} \simeq 2.5882, \nabla_{2}=0.91463, \nabla_{3} \simeq 0.25392
\end{aligned}
$$

and

$$
\begin{aligned}
& \Delta_{1} \simeq-4.8983 \times 10^{-3}, \Delta_{2} \simeq-2.243 \times 10^{-2}, \\
& \Delta_{3} \simeq-1.177 \times 10^{-2}, \Delta_{4} \simeq-3.4429 \times 10^{-2} \\
& \Pi_{2}=\Delta_{1} \Delta_{4}-\Delta_{2} \Delta_{3} \simeq-9.5358 \times 10^{-5} \\
& \Phi_{1} \simeq 2.1432, \Phi_{2}=0.40770, \Phi_{3} \simeq 0.59613
\end{aligned}
$$

So, for $t \in[1, e]$ and $\left(u_{1}, v_{1}\right),\left(u_{2}, v_{2}\right) \in \mathbb{R}^{2}$, we have

$$
\begin{aligned}
\left|f\left(t, u_{1}, v_{1}\right)-f\left(t, u_{2}, v_{2}\right)\right| & \leq \frac{1}{\left(25 \pi^{2}+t\right)}\left(\left|u_{1}-u_{2}\right|+\left|v_{1}-v_{2}\right|\right), \\
\left|g\left(t, u_{1}, v_{1}\right)-g\left(t, u_{2}, v_{2}\right)\right| & \leq \frac{1}{57(t+1)^{2}}\left(\left|u_{1}-u_{2}\right|+\left|v_{1}-v_{2}\right|\right), \\
\left|\varphi\left(t, u_{1}, v_{1}\right)-\varphi\left(t, u_{2}, v_{2}\right)\right| & \leq \frac{1}{55 t^{2}+1}\left(\left|u_{1}-u_{2}\right|+\left|v_{1}-v_{2}\right|\right), \\
\left|g_{2}\left(t, u_{1}, v_{1}\right)-g_{2}\left(t, u_{2}, v_{2}\right)\right| & \leq \frac{1}{49\left(t^{2}+2\right)}\left(\left|u_{1}-u_{2}\right|+\left|v_{1}-v_{2}\right|\right) .
\end{aligned}
$$


It follows that

$$
k_{1}=\frac{1}{\left(25 \pi^{2}+e\right)}, k_{2}=\frac{1}{140}, l_{1}=\frac{1}{55 e^{2}+1}, l_{2}=\frac{1}{49\left(e^{2}+2\right)} .
$$

Then,

$$
k_{1} \nabla_{1}+k_{2} \nabla_{2}+\nabla_{3}+l_{1} \Phi_{1}+l_{2} \Phi_{2}+\Phi_{3} \approx 0.97676<1 .
$$

By Theorem 2.1, we conclude that the system 20]. has a unique solution on $[1, e]$.

\section{Existence result}

We show the existence of solutions for the system (1)-(2) by applying Lemma 1.2.

For the forthcoming result, we impose the following conditions:

$\left(C_{3}\right): f, \varphi:[1, e] \times \mathbb{R} \times \mathbb{R} \rightarrow \mathbb{R}$, are continuous functions and there exist real constants $\omega_{1}>0, \varpi_{1}>0$ such that for any $t \in[1, e]$ and $u, v \in \mathbb{R}$, we have

$$
|f(t, u(t), v(t))| \leq \omega_{1}, \quad|\varphi(t, u(t), v(t))| \leq \omega_{2} .
$$

$\left(C_{4}\right): g, \psi:[1, e] \times \mathbb{R} \times \mathbb{R} \rightarrow \mathbb{R}$, are continuous functions and there exist real constants $\omega_{2}>0, \varpi_{2}>0$ such that for all $u, v \in \mathbb{R}$ and $t \in[1, e]$, we have

$$
|g(t, u(t), v(t))| \leq \varpi_{1}, \quad|\psi(t, u(t), v(t))| \leq \varpi_{2} .
$$

Theorem 3.1. Assume that hypotheses $\left(C_{3}\right)$ and $\left(C_{4}\right)$ hold. Furthermore, assume that $\Pi_{1} \neq 0$ and $\Pi_{2} \neq 0$. Then, system (1)-(2) has at least one solution.

Proof. By continuity of functions of $f, g \varphi$ and $\psi$, the operator $H$ is continuous.

Now, we show that the operator $H$ is completely continuous.

(I) First, we show that $H$ maps bounded sets of $W$ into bounded sets of $W$. Let us take $\varepsilon>0$ and $B_{\varepsilon}=\left\{(u, v) \in W:\|u, v\|_{W} \leq \varepsilon\right\}$. Then for $(u, v) \in B_{\varepsilon}$, we have

$$
\begin{aligned}
& \left\|H_{1}(u, v)\right\| \\
\leq & \sup _{t \in[1, e]}\left\{I^{\alpha_{1}+\beta_{1}}|f(s, u(s), v(s))|(t)+I^{\alpha_{1}+\beta_{1}+\theta_{1}}|\psi(s, u(s), v(s))|(t)+\left|\lambda_{1}\right| I^{\alpha_{1}}|v(s)|(t)\right. \\
& +\frac{\left|\Lambda_{2}\right|(\log t)^{\alpha_{1}}+\left|\Lambda_{1}\right| \Gamma\left(\alpha_{1}+1\right)}{\left|\Pi_{1}\right| \Gamma\left(\alpha_{1}+1\right)}\left(\left|\gamma_{1}\right|+|a| I^{p_{1}+\alpha_{1}+\beta_{1}}|f(s, u(s), v(s))|\left(\eta_{1}\right)\right. \\
& \left.+|a| I^{p_{1}+\alpha_{1}+\beta_{1}+\theta_{1}}|\varphi(s, u(s), v(s))|\left(\eta_{1}\right)+\left|\lambda_{1}\right|\left|a_{1}\right| I^{p_{1}+\alpha_{1}}|u(s)|\left(\eta_{1}\right)\right) \\
& +\frac{\left|\Lambda_{4}\right|(\log t)^{\alpha_{1}}+\left|\Lambda_{3}\right| \Gamma\left(\alpha_{1}+1\right)}{\left|\Pi_{1}\right| \Gamma\left(\alpha_{1}+1\right)}\left(\left|\gamma_{2}\right|+|b| I^{q_{1}+\alpha_{1}+\beta_{1}}\left|f_{1}(s, u(s), v(s))\right|\left(\xi_{1}\right)\right. \\
& \left.\left.+|b| I^{q_{1}+\alpha_{1}+\beta_{1}+\theta_{1}}|\varphi(s, u(s), v(s))|\left(\xi_{1}\right)+\left|\lambda_{1}\right||b| I^{q_{1}+\alpha_{1}}|u(s)|\left(\xi_{1}\right)\right)\right\}
\end{aligned}
$$


By $\left(C_{3}\right)$, we obtain

$$
\begin{aligned}
& \left\|H_{1}(u, v)\right\| \\
\leq & {\left[\frac{1}{\Gamma\left(\alpha_{1}+\beta_{1}+1\right)}+\frac{|a|\left(\left|\Lambda_{2}\right|+\left|\Lambda_{1}\right| \Gamma\left(\alpha_{1}+1\right)\right)\left(\log \eta_{1}\right)^{p_{1}+\alpha_{1}+\beta_{1}}}{\left|\Pi_{1}\right| \Gamma\left(\alpha_{1}+1\right) \Gamma\left(p_{1}+\alpha_{1}+\beta_{1}+1\right)}\right.} \\
& \left.+\frac{|b|\left(\left|\Lambda_{4}\right|+\left|\Lambda_{3}\right| \Gamma\left(\alpha_{1}+1\right)\right)\left(\log \xi_{1}\right)^{q_{1}+\alpha_{1}+\beta_{1}}}{\left|\Pi_{1}\right| \Gamma\left(\alpha_{1}+1\right) \Gamma\left(q_{1}+\alpha_{1}+\beta_{1}+1\right)}\right] \omega_{1} \\
& +\left[\frac{1}{\Gamma\left(\alpha_{1}+\beta_{1}+\theta_{1}+1\right)}+\frac{|a|\left(\left|\Lambda_{2}\right|+\left|\Lambda_{1}\right| \Gamma\left(\alpha_{1}+1\right)\right)\left(\log \eta_{1}\right)^{p_{1}+\alpha_{1}+\beta_{1}+\theta_{1}}}{\left|\Pi_{1}\right| \Gamma\left(\alpha_{1}+1\right) \Gamma\left(p_{1}+\alpha_{1}+\beta_{1}+\theta_{1}+1\right)}\right. \\
& \left.+\frac{|b|\left(\left|\Lambda_{4}\right|+\left|\Lambda_{3}\right| \Gamma\left(\alpha_{1}+1\right)\right)\left(\log \xi_{1}\right)^{q_{1}+\alpha_{1}+\beta_{1}+\theta_{1}}}{\left|\Pi_{1}\right| \Gamma\left(\alpha_{1}+1\right) \Gamma\left(q_{1}+\alpha_{1}+\beta_{1}+\theta_{1}+1\right)}\right] \omega_{2} \\
& +\left|\lambda_{1}\right|\left[\frac{1}{\Gamma\left(\alpha_{1}+1\right)}+\frac{|a|\left(\left|\Lambda_{2}\right|+\left|\Lambda_{1}\right| \Gamma\left(\alpha_{1}+1\right)\right)\left(\log \eta_{1}\right)^{p_{1}+\alpha_{1}}}{\left|\Pi_{1}\right| \Gamma\left(\alpha_{1}+1\right) \Gamma\left(p_{1}+\alpha_{1}+1\right)}\right. \\
& \left.+\frac{\left|b_{1}\right|\left(\left|\Lambda_{4}\right|+\left|\Lambda_{3}\right| \Gamma\left(\alpha_{1}+1\right)\right)\left(\log \xi_{1}\right)^{q_{1}+\alpha_{1}}}{\left|\Pi_{1}\right| \Gamma\left(\alpha_{1}+1\right) \Gamma\left(q_{1}+\alpha_{1}+1\right)}\right] \varepsilon \\
& +\frac{1}{\left|\Pi_{1}\right| \Gamma\left(\alpha_{1}+1\right)}\left[\left|\Delta_{2}\right|+\left|\Delta_{1}\right| \Gamma\left(\alpha_{1}+1\right)\left|\gamma_{1}\right|+\left|\Delta_{4}\right|+\left|\Delta_{3}\right| \Gamma\left(\alpha_{1}+1\right)\left|\gamma_{2}\right|\right] \\
= & \nabla_{1} \omega_{1}+\nabla_{2} \omega_{2}+\nabla_{3} \varepsilon+\nabla_{4},
\end{aligned}
$$

which implies that

$$
\left\|H_{1}(u, v)\right\| \leq \nabla_{1} \omega_{1}+\nabla_{2} \omega_{2}+\nabla_{3} \varepsilon+\nabla_{4} .
$$

As before, it can be shown that

$$
\left\|H_{2}(u, v)\right\| \leq \Phi_{1} \varpi_{1}+\Phi_{2} \varpi_{2}+\Phi_{3} \varepsilon+\Phi_{4} .
$$

It follows from $(21)$ and $(22)$ that

$$
\begin{aligned}
& \|H(u, v)\|_{W} \\
\leq & \nabla_{1} \omega_{1}+\nabla_{2} \omega_{2}+\nabla_{3} \varepsilon+\nabla_{4}+\Phi_{1} \varpi_{1}+\Phi_{2} \varpi_{2}+\Phi_{3} \varepsilon+\Phi_{4}<\infty .
\end{aligned}
$$

$(I I)$ Next, we show that $H$ is equicontinuous. Let $(u, v) \in B_{\varepsilon}$ and $t_{1}, t_{2} \in[1, e]$ with $t_{1}<t_{2}$. Then we have

$$
\begin{aligned}
& \left|H_{2}(u, v)\left(t_{2}\right)-H_{2}(u, v)\left(t_{1}\right)\right| \\
\leq & \left.\frac{\omega_{1}}{\Gamma\left(\alpha_{1}+\beta_{1}\right)}\left(\mid \log t_{2}\right)^{\alpha_{1}+\beta_{1}}-\left(\log t_{1}\right)^{\alpha_{1}+\beta_{1}}|+|\left(\log t_{2}-\log t_{1}\right)^{\alpha_{1}+\beta_{1}} \mid\right) \\
& \left.+\frac{\omega_{2}}{\Gamma\left(\alpha_{1}+\beta_{1}+\theta_{1}\right)}\left(\mid \log t_{2}\right)^{\alpha_{1}+\beta_{1}+\theta_{1}}-\left(\log t_{1}\right)^{\alpha_{1}+\beta_{1}+\theta_{1}}|+|\left(\log t_{2}-\log t_{1}\right)^{\alpha_{1}+\beta_{1}+\theta_{1}} \mid\right) \\
& +\frac{\varepsilon\left|\lambda_{1}\right|}{\Gamma\left(\alpha_{1}\right)}\left(\left|\left(\log t_{2}\right)^{\alpha_{1}}-\left(\log t_{1}\right)^{\alpha_{1}}\right|+\left|\left(\log t_{2}-\log t_{1}\right)^{\alpha_{1}}\right|\right) \\
& +\frac{\left|\Lambda_{2}\right|\left|\left(\log t_{2}\right)^{\alpha_{1}}-\left(\log t_{1}\right)^{\alpha_{1}}\right|}{\left|\Pi_{1}\right| \Gamma\left(\alpha_{1}+1\right)}\left(\left|\gamma_{1}\right|+\frac{\omega_{1}|a|\left(\log \eta_{1}\right)^{p_{1}+\alpha_{1}+\beta_{1}}}{\Gamma\left(p_{1}+\alpha_{1}+\beta_{1}\right)}\right. \\
& \left.+\frac{\omega_{2}|a|\left(\log \eta_{1}\right)^{p_{1}+\alpha_{1}+\beta_{1}+\theta_{1}}}{\Gamma\left(p_{1}+\alpha_{1}+\beta_{1}+\theta_{1}\right)}+\frac{\varepsilon\left|\lambda_{1}\right||a|\left(\log \eta_{1}\right)^{p_{1}+\alpha_{1}}}{\Gamma\left(p_{1}+\alpha_{1}\right)}\right) \\
& +\frac{\left|\Lambda_{4}\right|\left|\left(\log t_{2}\right)^{\alpha_{1}}-\left(\log t_{1}\right)^{\alpha_{1}}\right|}{\left|\Pi_{1}\right| \Gamma\left(\alpha_{1}+1\right)}\left(\left|\gamma_{2}\right|+\frac{\omega_{1}|b|\left(\log \xi_{1}\right)^{q_{1}+\alpha_{1}+\beta_{1}}}{\Gamma\left(q_{1}+\alpha_{1}+\beta_{1}\right)}\right. \\
& \left.+\frac{\omega_{2}|b|\left(\log \xi_{1}\right)^{q_{1}+\alpha_{1}+\beta_{1}+\theta_{1}}}{\Gamma\left(q_{1}+\alpha_{1}+\beta_{1}+\theta_{1}\right)}+\frac{\varepsilon\left|\lambda_{1}\right||b|\left(\log \xi_{1}\right)^{q_{1}+\alpha_{1}}}{\Gamma\left(q_{1}+\alpha_{1}\right)}\right)
\end{aligned}
$$


which imply that $\left\|H_{1}(u, v)\left(t_{2}\right)-H_{1}(u, v)\left(t_{1}\right)\right\| \rightarrow 0$ independent of $u$ and $v$ as $t_{2} \rightarrow t_{1}$. Analogously, we obtain

$$
\begin{aligned}
& \left|H_{1}(u, v)\left(t_{2}\right)-H_{1}(u, v)\left(t_{1}\right)\right| \\
\leq & \left.\frac{\varpi_{1}}{\Gamma\left(\alpha_{2}+\beta_{2}\right)}\left(\mid \log t_{2}\right)^{\alpha_{2}+\beta_{2}}-\left(\log t_{1}\right)^{\alpha_{2}+\beta_{2}}|+|\left(\log t_{2}-\log t_{1}\right)^{\alpha_{2}+\beta_{2}} \mid\right) \\
& \left.+\frac{\varpi_{2}}{\Gamma\left(\alpha_{2}+\beta_{2}+\theta_{2}\right)}\left(\mid \log t_{2}\right)^{\alpha_{2}+\beta_{2}+\theta_{2}}-\left(\log t_{1}\right)^{\alpha_{2}+\beta_{2}+\theta_{2}}|+|\left(\log t_{2}-\log t_{1}\right)^{\alpha_{2}+\beta_{2}+\theta_{2}} \mid\right) \\
& +\frac{\varepsilon\left|\lambda_{2}\right|}{\Gamma\left(\alpha_{2}\right)}\left(\left|\left(\log t_{2}\right)^{\alpha_{2}}-\left(\log t_{1}\right)^{\alpha_{2}}\right|+\left|\left(\log t_{2}-\log t_{1}\right)^{\alpha_{2}}\right|\right) \\
& +\frac{\left|\Delta_{2}\right|\left|\left(\log t_{2}\right)^{\alpha_{2}}-\left(\log t_{1}\right)^{\alpha_{2}}\right|}{\left|\Pi_{2}\right| \Gamma\left(\alpha_{2}+1\right)}\left(\left|\sigma_{1}\right|+\frac{\varpi_{1}|c|\left(\log \eta_{2}\right)^{p_{2}+\alpha_{2}+\beta_{2}}}{\Gamma\left(p_{2}+\alpha_{2}+\beta_{2}\right)}\right. \\
& \left.+\frac{\varpi_{2}|c|\left(\log \eta_{2}\right)^{p_{2}+\alpha_{2}+\beta_{2}+\theta_{2}}}{\Gamma\left(p_{2}+\alpha_{2}+\beta_{2}+\theta_{2}\right)}+\frac{\varepsilon\left|\lambda_{2}\right||c|\left(\log \eta_{2}\right)^{p_{2}+\alpha_{2}}}{\Gamma\left(p_{2}+\alpha_{2}\right)}\right) \\
& +\frac{\left|\Delta_{4}\right|\left|\left(\log t_{2}\right)^{\alpha_{2}}-\left(\log t_{1}\right)^{\alpha_{2}}\right|}{\left|\Pi_{2}\right| \Gamma\left(\alpha_{2}+1\right)}\left(\left|\sigma_{2}\right|+\frac{\varpi_{1}|d|\left(\log \xi_{2}\right)^{q_{2}+\alpha_{2}+\beta_{2}}}{\Gamma\left(q_{2}+\alpha_{2}+\beta_{2}\right)}\right. \\
& \left.+\frac{\varpi_{2}|d|\left(\log \xi_{2}\right)^{q_{2}+\alpha_{2}+\beta_{2}+\theta_{2}}}{\Gamma\left(q_{2}+\alpha_{2}+\beta_{2}+\theta_{2}\right)}+\frac{\varepsilon\left|\lambda_{2}\right||d|\left(\log \xi_{2}\right)^{q_{2}+\alpha_{2}}}{\Gamma\left(q_{2}+\alpha_{2}\right)}\right)
\end{aligned}
$$

which imply that $\left\|H_{2}(u, v)\left(t_{2}\right)-H_{2}(u, v)\left(t_{1}\right)\right\| \rightarrow 0$ independent of $u$ and $v$ as $t_{2} \rightarrow t_{1}$. Therefore, the operator $H(u, v)$ is equicontinuous. Combining $(I)$ and $(I I)$ and using the Arzelà-Ascoli theorem, we conclude that $H$ is completely continuous operator.

(III)Finally, it will be verified that the $\Omega=\{(u, v) \in W,(u, v)=\mu H(u, v), 0<\mu<1\}$, is bounded. Let $(u, v) \in \Omega$, then $(u, v)=\mu H(u, v)$, for some $0<\mu<1$. Hence, for $t \in[1, e]$, we have

$$
u(t)=\mu H_{1}(u, v)(t), v(t)=\mu H_{2}(u, v)(t) .
$$

By $\left(C_{3}\right)$ and $\left(C_{4}\right)$, we have

$$
\|u\| \leq \mu\left(\nabla_{1} \omega_{1}+\nabla_{2} \omega_{2}+\nabla_{3} \varepsilon+\nabla_{4}\right)
$$

and

$$
\|v\| \leq \mu\left(\Phi_{1} \varpi_{1}+\Phi_{2} \varpi_{2}+\Phi_{3} \varepsilon+\Phi_{4}\right) .
$$

It follows from $(23)$ and $(24)$, that

$$
\begin{aligned}
& \|(u, v)\|_{W} \\
\leq & \mu\left[\nabla_{1} \omega_{1}+\nabla_{2} \omega_{2}+\nabla_{3} \varepsilon+\nabla_{4},+\Phi_{1} \varpi_{1}+\Phi_{2} \varpi_{2}+\Phi_{3} \varepsilon+\Phi_{4}\right] \\
\leq & \nabla_{1} \omega_{1}+\nabla_{2} \omega_{2}+\nabla_{3} \varepsilon+\nabla_{4},+\Phi_{1} \varpi_{1}+\Phi_{2} \varpi_{2}+\Phi_{3} \varepsilon+\Phi_{4} .
\end{aligned}
$$

Consequently,

$$
\|(u, v)\|_{W}<\infty
$$

This shows that the set is bounded.

Thanks to $(I),(I I)$ and $(I I I)$, and by Lemma 1.2, we deduce that $H$ has at least one fixed point, which is a solution of system (1)-(2). 
Example 3.1. For $t \in[1, e]$, consider the following system

$$
\left\{\begin{array}{c}
D^{\frac{27}{44}}\left(D^{\frac{16}{25}}+\frac{1}{49}\right) u(t)-\frac{2 \ln (t)|\sin u(t)|}{t^{2}+1}-\frac{|v(t)|(3 t-e)}{4 e^{t}(|y(t)|+1)}=I^{\frac{1}{2}}\left(\frac{|u(t)+v(t)|(3-t)^{2}}{\sqrt{t+8}(|u(t)|+|v(t)|+1)}\right), \\
D^{\frac{13}{20}}\left(D^{\frac{8}{11}}-\frac{1}{33}\right) y(t)-\frac{e^{-|u(t)-v(t)|}+7}{(t+3)^{3}}=I^{\frac{21}{40}}\left(\frac{|\cos u(t)|+e^{t-e}}{5 t^{2}+\ln t-2}+\frac{|\sin v(t)|}{3 t^{4}-1}\right) \\
4 I^{\frac{17}{50}} u\left(\frac{38}{25}\right)=(\log 8-\log 5)^{-\frac{1}{2}}, 3 I^{\frac{9}{10}} u\left(\frac{8}{3}\right)=6(\log 8-\log 3)^{-\frac{1}{10}} \\
\frac{3}{2} I^{\frac{1}{3}} v\left(\frac{8}{5}\right)=2(\log 8-\log 5)^{-\frac{2}{3}}, \frac{7}{2} I^{\frac{23}{25}} v\left(\frac{5}{2}\right)=\frac{21}{2}(\log 5-\log 2)^{-\frac{2}{25}}
\end{array}\right.
$$

We have

$$
\begin{aligned}
& f(t, u, v)=\frac{2 \ln (t)|\sin u|}{t^{2}+1}+\frac{(3 t-e)|v|}{4 e^{t}(|y(t)|+1)}, \varphi(t, u, v)=\frac{|u+v|(3-t)^{2}}{\sqrt{t+8}(|u|+|v|+1)}, \\
& g(t, u, v)=\frac{e^{-|u-v|}+7}{(t+3)^{3}}, \psi(t, u, v)=\frac{|\cos u|+e^{t-e}}{5 t^{2}+\ln t-2}+\frac{|\sin v|}{3 t^{4}-1} .
\end{aligned}
$$

and

$$
\begin{aligned}
& \Lambda_{1} \simeq-1.7185, \Lambda_{2} \simeq-3.3346, \Lambda_{3} \simeq-2.1289, \Lambda_{4} \simeq-3.0654 \\
& \Delta_{1} \simeq 0.31790, \Delta_{2} \simeq 0.63322, \Delta_{3} \simeq 2.0448, \Delta_{4} \simeq 3.3336
\end{aligned}
$$

We remark that

$$
\begin{aligned}
& \Pi_{1}=\Lambda_{1} \Lambda_{4}-\Lambda_{2} \Lambda_{3} \simeq-1.8311, \\
& \Pi_{2}=\Delta_{1} \Delta_{4}-\Delta_{2} \Delta_{3} \simeq-0.23506,
\end{aligned}
$$

and

$$
f(t, u, v) \leq \frac{3}{2}, \varphi(t, u, v) \leq \frac{(e-3)^{2}}{3}, g(t, u, v) \leq \frac{1}{8}, \psi(t, u, v) \leq \frac{7}{6} .
$$

So, by Theorem 3.1, system (25) has at least one solution on $[1, e]$.

\section{References}

[1] S. Aljoudi, B. Ahmad and A. Alsaedi, Existence and uniqueness results for a coupled system of Caputo-Hadamard fractional differential equations with nonlocal Hadamard type integral boundary conditions, Fractal Fract. 4(1), (2020) 1-15.

[2] A.Ardjouni, A.Djoudi, Existence and uniqueness of solutions for nonlinear implicit Caputo-Hadamard fractional differential equations with nonlo cal conditions, Adv.Theory Nonlinear Anal. Appl. 3(1) (2019), 46-52.

[3] M. Benchohra, M. Slimane, Fractional differential inclusions with non instantaneous impulses in Banach spaces, Rsults in Nonlinear Anal. 2(1) (2019), 36-47.

[4] W. Benhamida, S. Hamani and J. Henderson. Boundary value problems for Caputo-Hadamard fractional differential equations, Adv.Theory Nonlinear Anal. Appl. 2 (3), (2018), 138-145.

[5] Z. Dahmani, L. Tabharit. Fractional order differential equations involving Caputo derivative. Theory Appl. Math. Comput. Sci. 4(1) (2014), 40-55.

[6] S. Hamani , W. Benhamida and J. Henderson , Boundary value problems for Caputo-Hadamard fractional differential equations, Adv.TheoryNonlinear Anal. Appl. 2(3) (2018) 138-145.

[7] A.A. Hamoud, Existence and uniqueness of solutions for fractional neutral Volterra-Fredholm integro differential equations. Adv.Theory Nonlinear Anal. Appl. 4(4) (2019), 162-173.

[8] M. Houas, K.O. Melha, Existence and uniqueness results for a coupled system of Hadamard fractional differential equations with multi-point boundary conditions. Facta. Univ. Ser. Math. Inform. 35(3) (2020), 843-856.

[9] M. Houas, Existence and uniqueness results for a coupled system of nonlinear fractional differential equations with two fractional orders, J. Interdisciplinary Mathematics. 23(6) (2020), 1047-1064.

[10] M. Houas, Existence of solutions for nonlinear Hadamard differential equations with nonlocal conditions, J. Interdisciplinary Mathematics. 24(3) (2021), 593-612.

[11] M. Houas. Existence of solutions for fractional differential equations involving two Riemann-Liouville fractional orders. Anal. Theory Appl. 34(3), (2018), 253-274. 
[12] A.A. Kilbas, H.M. Srivastava, J.J.Trujillo. Theory and applications of fractional differential equations. North-Holland Mathematics Studies. 204. Elsevier Science B.V. Amsterdam. 2006.

[13] D. Luo, A. Zada, S. Shaleena and M. Ahmad, Analysis of a coupled system of fractional differential equations with non-separated boundary conditions, Adv. Differ. Equa. 2020: 590 (2020), 1-24.

[14] K. Malara, A. Anguraj, Existence results for fractional integrodifferential systems with interval impulse via sectorial operator, Rsults in Nonlinear Anal. 2(4) (2019) 169-181.

[15] S. Muthaiaha, M. Murugesana and N.G.Thangaraja, Existence of solutions for nonlocal boundary value problem of Hadamard fractional differential equations, Adv.Theory Nonlinear Anal. Appl. 3(3) (2019), 162-173.

[16] S.K. Ntouyas and J. Tariboon. Fractional integral problems for Hadamard-Caputo fractional Langevin differential inclusions. J. Appl. Math. Comput. 51, (2016), 13-33.

[17] A.Taieb and Z. Dahmami. A coupled system of nonlinear differential equations involving $m$ nonlinear terms. Georgian Math. J. 23(3), 2016, 447-458.

[18] P. Thiramanus, S.K. Ntouyas and J. Tariboon. Positive solutions for Hadamard fractional differential equations on infinite domain. Adv. Difference. Equ. 83 (2016), 1-18.

[19] W. Yang Y. Qinc. Positive solutions for nonlinear Hadamard fractional differential equations with integral boundary conditions. Science Asia. 43 (2017), 201-206.

[20] G. Wang, W. Liu, C. Ren. Existence of solutions for multi-point nonlinear differential equations of fractional orders with integral boundary conditions. Elec. J. Diff. Equ. 54 (2012), 1-10.

[21] W. Yang. Positive solutions for a coupled system of nonlinear fractional differential equations with integral boundary conditions. Comput. Math. Appl. 63(1) (2012), 288-297. 